\title{
The effect of warm gas on the buckling instability in galactic bars
}

\author{
Ewa L. Łokas
}

\begin{abstract}
Nicolaus Copernicus Astronomical Center, Polish Academy of Sciences, Bartycka 18, 00-716 Warsaw, Poland e-mail: lokas@camk.edu.pl
\end{abstract}

Received 21 November 2019 / Accepted 5 January 2020

\begin{abstract}
By using $N$-body and hydro simulations, we study the formation and evolution of bars in galaxies with significant gas content focusing on the phenomenon of the buckling instability. The galaxies are initially composed of a spherical dark matter halo and only stellar, or stellar and gaseous, disks with parameters that are similar to the Milky Way and are evolved for $10 \mathrm{Gyr}$. We consider different values of the gas fraction $f=0-0.3$ and in order to isolate the effect of the gas, we kept the fraction constant during the evolution by not allowing the gas to cool and form stars. The stellar bars that form in simulations with higher gas fractions are weaker and shorter, and they do not form at all for gas fractions that are higher than 0.3. The bar with a gas fraction of 0.1 forms sooner due to initial perturbations in the gas, but despite the longer evolution, it does not become stronger than the one in the collisionless case at the end of evolution. The bars in the gas component are weaker; they reach their maximum strength around $4 \mathrm{Gyr}$ and later decline to transform into spheroidal shapes. The distortion of the stellar bar during the buckling instability is weaker for higher gas fractions and weakens the bar less significantly, but it has a similar structure both in terms of radial profiles and in face-on projections. For $f=0.2$, the first buckling lasts significantly longer and the bar does not undergo the secondary buckling event, while for $f=0.3$, the buckling does not occur. Despite these differences, all bars develop boxy/peanut shapes in the stellar and gas component by the end of the evolution, although their thickness is smaller for higher gas fractions.
\end{abstract}

Key words. galaxies: evolution - galaxies: fundamental parameters - galaxies: kinematics and dynamics - galaxies: spiral galaxies: structure

\section{Introduction}

A significant fraction of late-type galaxies in the Universe is barred (Knapen et al. 2000; Aguerri et al. 2009; Nair \& Abraham 2010; Buta et al. 2015; Erwin 2018). This morphological feature has been studied over the last few decades both observationally and theoretically, mainly via computer simulations. It has been established that bars can form via inherent instability of galactic disks (Hohl 1971; Ostriker \& Peebles 1973; Sellwood 1981) when evolving in isolation and they grow by transferring angular momentum from the disk to the halo (Athanassoula 2003). Bars can also be induced in disks that are stable in isolation if they are subject to strong enough tidal forces from neighboring structures (Noguchi 1996; Łokas et al. 2014, 2016; Gajda et al. 2017; Łokas 2018).

A common episode in the evolution of strong bars, independent of their origin, is the phenomenon of buckling instability. The instability, which was first discussed by Combes \& Sanders (1981), Pfenniger \& Friedli (1991), and Raha et al. (1991), manifests itself by noticeable distortions of the bar out of the disk plane that modify the orbital structure of the bar producing a pronounced boxy/peanut shape of the bar when seen edge-on, which is quite similar to those observed (Erwin \& Debattista 2017). The first rather short buckling event occurs in the inner part of the bar and can also then extend itself to the outer region where it lasts much longer (Martinez-Valpuesta et al. 2006).

The nature of the instability remains controversial; it has been ascribed to the so-called fire-hose instability and is then supposed to be triggered by a low enough ratio of the vertical to horizontal velocity dispersion of the stars in the bar (Toomre 1966; Merritt \& Hernquist 1991; Merritt \& Sellwood 1994). Another possibility, first proposed by Combes et al. (1990) and Pfenniger \& Friedli (1991), and more recently by Quillen (2002), is that the instability results from trapping $\mathrm{x} 1$ orbits of the bar at vertical resonances. However, these resonances should mainly apply to banana-like orbits, which are known to only contribute a little to the final boxy/peanut shape (Portail et al. 2015; Abbott et al. 2017).

In Łokas (2019a), by using simulations of tidally induced bars, we demonstrate that there is no direct relation between the ratio of the vertical to horizontal velocity dispersion and the occurrence of buckling, which is in opposition to the interpretation of buckling as the fire-hose instability. In Łokas (2019b) we study the buckling instability in a collisionless simulation of a Milky Way-like galaxy, looking in particular at the evolution of the orbital structure of the bar due to buckling. It turns out that buckling seems to be indeed initiated by the vertical instability of $\mathrm{x} 1$ orbits that cause the initial distortion of the bar out of the disk plane. This distortion then evolves as kinematic bending waves changing the orbital structure into a combination of much more complicated orbits; however, they all obey a very tight relation between the vertical and circular frequency.

The effect of the dissipative component of galaxies on the evolution of bars, and the buckling instability in particular, has so far been studied mainly in the context of the discussion concerning the bar survival or longevity. The controversy started with the results of Bournaud \& Combes (2002) and Bournaud et al. (2005) who claimed, based on their simulations, that the bars in gas-rich disks are short-lived because of the formation of the central mass concentration and the torques between the stellar bar and the gaseous arms. Later studies (Debattista et al. 2006; Berentzen et al. 2007; Villa-Vargas et al. 2010; Athanassoula et al. 2013) disagreed with these conclusions and showed that bars can 
survive in galaxies with significant gas content and their temporary weakening is due to the buckling instability. These studies, however, demonstrated that bars are generally weaker in gasrich galaxies and indicated that buckling instability is then less pronounced.

In this paper, we revisit the issue of the buckling instability with the aim of isolating the effect of the presence of a significant fraction of warm gas in the disk. In order to control the gas content, it seems reasonable to keep it constant by not allowing it to cool and form stars. Otherwise, as demonstrated by simulations of Athanassoula et al. (2013), the gas is quickly depleted. They considered galaxies with gas fractions from a wide range initially, but these fractions, due to star formation, turned out to be quite similar at the most interesting time when the bar forms. While it may seem more realistic to include cooling, star formation, and feedback, these processes do not take into account the continuous supply of gas from the hot halo, which has been demonstrated to significantly affect the evolution of galaxies (Roškar et al. 2008). Given this, the assumption of a constant gas fraction may not be as unrealistic as it seems and it may reflect, to some extent, the actual gas content history in real galaxies. Our approach is thus more similar to the one of Berentzen et al. (2007) and Villa-Vargas et al. (2010) who considered constant gas fractions. However, in contrast to their studies, we used a higher resolution for the gas, a novel method to solve hydrodynamics, and evolved the gas adiabatically instead of keeping it isothermal. We also applied newly developed methods to quantify and visualize buckling instability.

The paper is organized in the following way. In Sect. 2 we present the simulations used in this study. Section 3 describes the formation and evolution of the bars and Sect. 4 gives a more detailed description of buckling in the presence of a dissipative component. The discussion follows in Sect. 5.

\section{Simulations}

For the purpose of this study, we ran a series of simulations of a galaxy similar to the Milky Way evolving in isolation. The initial conditions for each simulation were the same, except for the gas fraction in the disk. All galaxy models were initially composed of a spherical dark matter halo and a purely stellar exponential disk, in the collisionless case, or a stellar and a gaseous disk, in the remaining cases. The dark matter halo had a Navarro-FrenkWhite (Navarro et al. 1997) profile with a virial mass of $M_{\mathrm{H}}=$ $10^{12} M_{\odot}$ and a concentration of $c=25$. The total mass of the disk (whether purely stellar or composed of stars and gas) was $M_{\mathrm{D}}=$ $4.5 \times 10^{10} M_{\odot}$, its targeted scale-length was $R_{\mathrm{D}}=3 \mathrm{kpc}$ (for both components), and the thickness was $z_{\mathrm{D}}=0.42 \mathrm{kpc}$ (for the stars). The central value of the radial velocity dispersion for the stars was $\sigma_{R, 0}=120 \mathrm{~km} \mathrm{~s}^{-1}$. The initial circular velocity curve of the simulated galaxies is shown in Fig. 1.

For the models containing gas, the mass of the stellar disk was reduced, the mass of the gaseous disk was increased, and we considered different gas fractions $f$ of the disk, from 0 to 0.7 with a step of 0.1 . All models had $10^{6}$ dark matter particles in the halo (of mass $10^{6} M_{\odot}$ each) and $10^{6}$ particles in the disk. The masses of the stellar and gas particles were always the same and equal to $4.5 \times 10^{4} M_{\odot}$. So, for example, the disk with the gas fraction $f=0.1$ had $10^{5}$ gas particles and $9 \times 10^{5}$ stars.

The galaxies were initialized using the new version of the GalactICS code (Deg et al. 2019) that extends the procedures described in Widrow \& Dubinski (2005) and Widrow et al. (2008) to include gaseous disks. The code uses the potential method of Wang et al. (2010) to create gaseous disks close

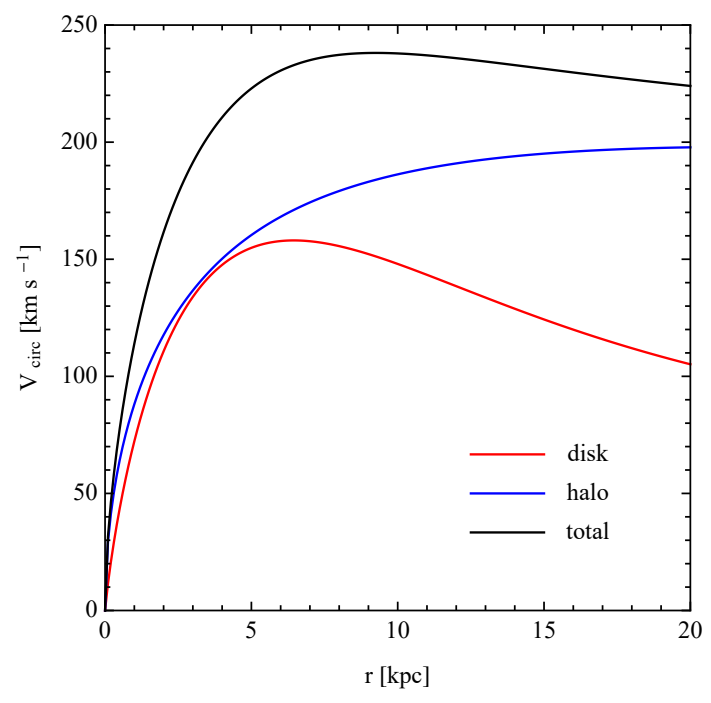

Fig. 1. Initial circular velocity curve of the simulated galaxies. The red, blue, and black curve corresponds to the disk, halo, and both components combined, respectively.

to equilibrium. The collisional component was assumed to be an ideal, adiabatic gas with a temperature of $10^{4} \mathrm{~K}$. The gas disks that were created were thin and flared toward larger radii, as expected for systems in equilibrium (Benítez-Llambay et al. 2018). To quantify the flaring, we estimated the median value of the vertical $|z|$ coordinates of the gas particles as a function of the cylindrical radius $R$. We find that the medians increase in radius with values of $0.03,0.06$, and $0.1 \mathrm{kpc}$ at $R=R_{\mathrm{D}}, 2 R_{\mathrm{D}}$, and $3 R_{\mathrm{D}}$, respectively, and they are very similar for all gas fractions. Instead, the analogous medians of $|z|$ for the stellar disks are almost constant with radius and are equal to $0.22-0.24 \mathrm{kpc}$ in the same range of radii.

The evolution of the galaxies was followed for $10 \mathrm{Gyr}$ with the GIZMO code (Hopkins 2015), an extension of the widely used GADGET-2 code (Springel et al. 2001; Springel 2005), saving outputs every 0.05 Gyr. The hydrodynamics was solved using the Lagrangian Meshless Finite-Mass (MFM) method, assuming the polytropic equation of state $(\gamma=5 / 3)$ for the gas. In order to keep the gas fraction constant in each simulation for the entire time, the gas was not allowed to cool or form stars so no prescriptions for cooling, star formation, or feedback were used. The softening scales were chosen following the recommendations in Hopkins et al. (2018) and they were fixed with $\epsilon_{\mathrm{D}}=12 \mathrm{pc}$ and $\epsilon_{\mathrm{H}}=75 \mathrm{pc}$ for the stellar disk and halo of the galaxy, respectively. For the gas, we used an adaptive gravitational softening with the minimum value of $\epsilon_{\mathrm{G}}=1 \mathrm{pc}$.

\section{Evolution of the bars}

The galaxies with a gas fraction of 0.4 and higher did not form a bar at all, so in the following analysis we restrict the discussion to the four simulations with gas fractions of $f=0,0.1,0.2$, and 0.3 . The face-on surface density distributions of the stars and gas in the disks at 4 and $10 \mathrm{Gyr}$ are shown in Figs. 2 and 3, respectively. In each of the figures, the plots in the left column are for the stars and those in the right one are for the gas, while subsequent rows correspond to an increasing gas fraction. We see that after $4 \mathrm{Gyr}$ of evolution (Fig. 2), the bars are already quite strong, both in the stellar and gaseous component. On the other hand, at the end of the simulations, after 10 Gyr (Fig. 3), 
E. L. Łokas: The effect of warm gas on the buckling instability in galactic bars
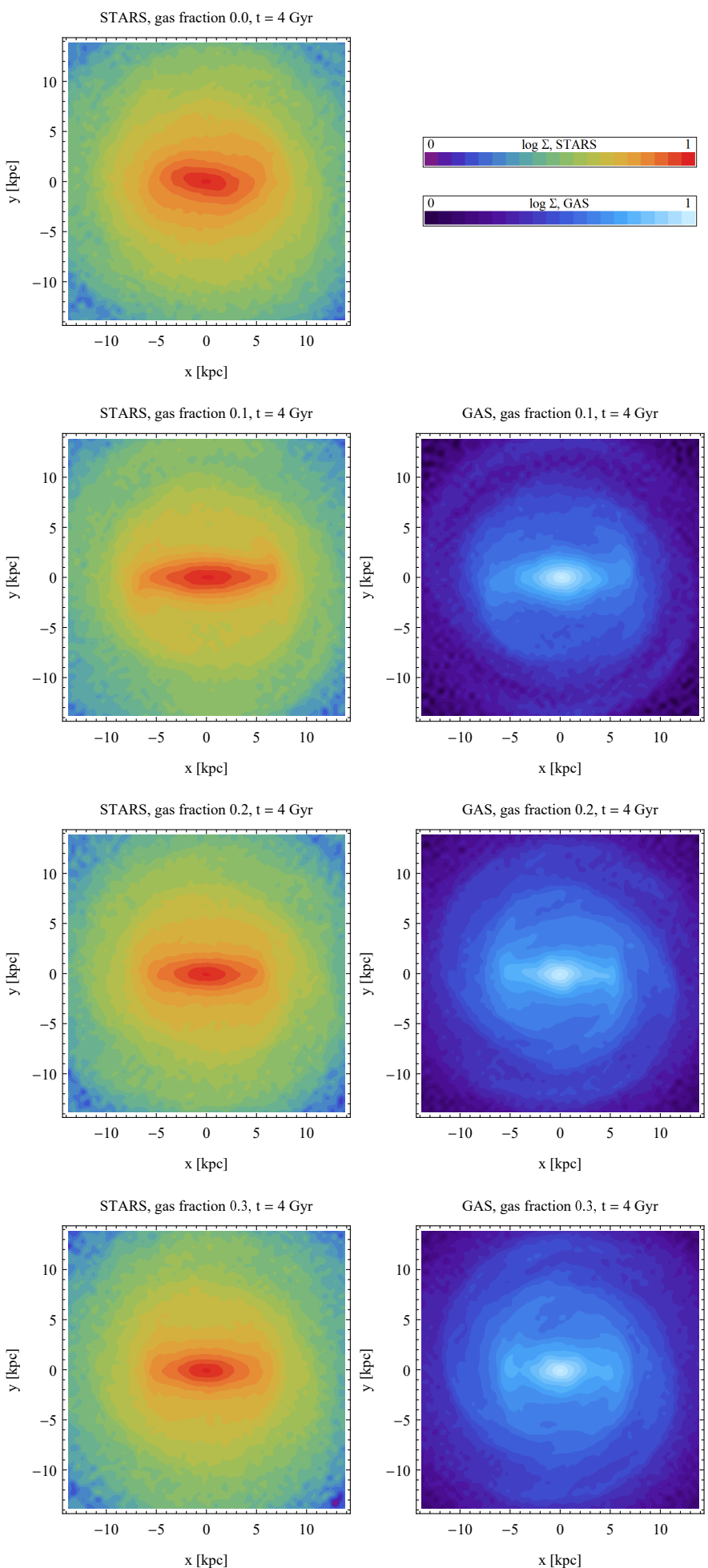

Fig. 2. Surface density distributions of stars and gas in the face-on view at $4 \mathrm{Gyr}$ from the start of the simulations when the gas bars are strongest Rows show the images from simulations with increasing gas fraction. Left column: stars, right column: gas. The surface densities were normalized to the central value in each case.

while the stellar bars become even stronger, the ones in the gas dissolve and only leave behind slightly oval shapes.

The simplest way to quantify the formation and evolution of the bar is to measure its strength as the $m=2$ mode of the Fourier decomposition of the surface distribution of particles projected along the short axis: $A_{m}(R)=\left|\Sigma_{j} \exp \left(\operatorname{im} \theta_{j}\right)\right| / N$. Here, $\theta_{j}$ is the
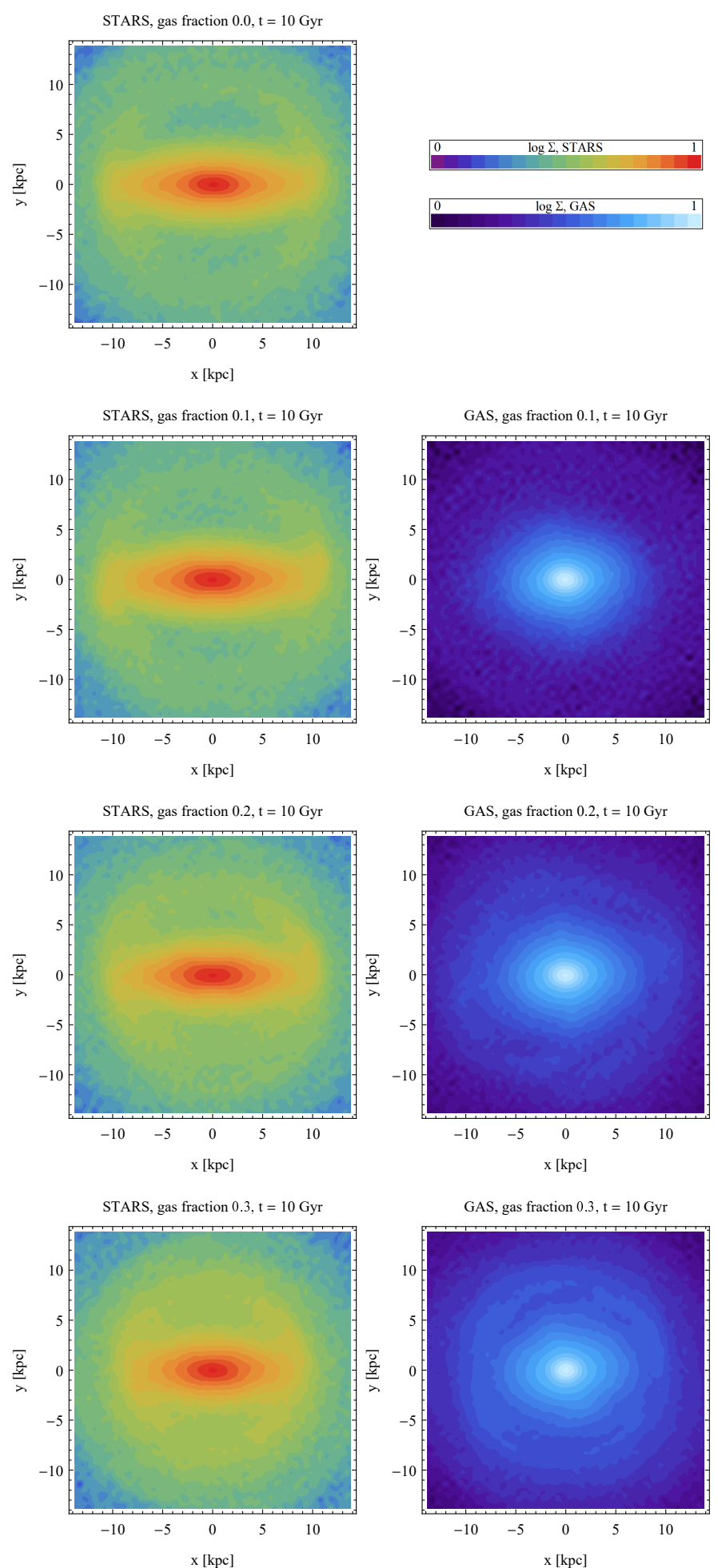

Fig. 3. Same as Fig. 2, but at the end of the simulations after $10 \mathrm{Gyr}$.

azimuthal angle of the $j$ th star and the sum is up to the total number of $N$ particles. The radius $R$ is the standard radius in cylindrical coordinates in the plane of the disk, $R=\left(x^{2}+y^{2}\right)^{1 / 2}$. Figure 4 shows this quantity measured for particles within twice the initial disk scale-length, $2 R_{\mathrm{D}}$. The upper panel of the figure shows the strength of the bar in stars and the lower one for the gas, with colors coding the gas fraction. For the gas fraction $f=0.4 A_{2}$ is always below 0.06 , both for stars and gas; we do not show this in the figure. We note that there is no specific trend in the stellar 

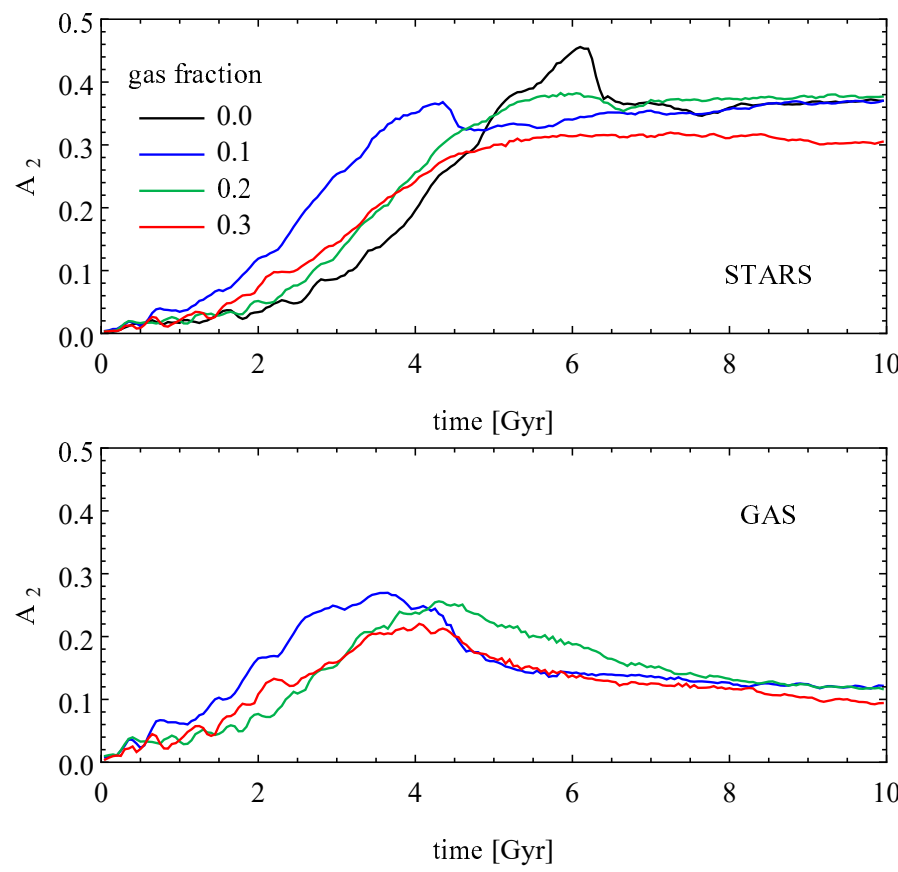

Fig. 4. Evolution of the bar mode $A_{2}$ for the stellar component (upper panel) and the gas (lower panel). The measurements were performed within $2 R_{\mathrm{D}}$, that is, the bar mode here is a single value $A_{2}=A_{2}\left(<2 R_{\mathrm{D}}\right)$. Lines of different colors correspond to different gas fractions.

bar formation time, that is, the galaxy with $f=0.1$ forms the bar first. This may be due to particular realizations of the initial conditions, which would be an example of stochasticity that is associated with bar formation and evolution (Sellwood \& Debattista 2009), although the presence of the gas may also play a role, as discussed below.

Figure 4 demonstrates that the stellar bar is the strongest in the purely stellar disk $(f=0)$ and the weakest in the high gas fraction case $(f=0.3)$. However, for the intermediate gas fractions ( $f=0.1$ and 0.2 ), the maximum strength of the bar is similar so there is no obvious monotonic dependence of the bar strength on the gas fraction. In addition, while the disks with gas fractions of $f=0,0.2$, and 0.3 reach the maximum bar strength after about $6 \mathrm{Gyr}$ of evolution, this maximum is reached much earlier (around $4.3 \mathrm{Gyr}$ ) for the low gas fraction case $(f=0.1)$. In spite of this, all stellar bars seem to end up with a similar strength at the end of the simulations, after $10 \mathrm{Gyr}$, except for the $f=0.3$ case, which has a slightly weaker bar.

All the bars with a gas fraction below 0.3 show a monotonic growth until they reach a maximum, after which the bar mode suddenly drops. This abrupt decrease of the bar strength is a clear signature of the buckling instability. We note that this drop is more pronounced the lower the gas fraction and it does not occur at all for $f=0.3$. This suggests that buckling does not happen in our highest gas fraction case.

The evolution of the bars in the gaseous component is significantly different, as demonstrated by the lower panel of Fig. 4. Again, the bar for $f=0.1$ grows the fastest, but all the bars reach maximum strength around $4 \mathrm{Gyr}$ after which time they start to decline slowly, while the stellar bars still grow or at least regain their strength after buckling. We also note that the time when the gaseous bars start to decline is not related to the time of buckling of the stellar bar. While for $f=0.1$ these times coincide, for $f=0.2$ the gas bar starts to decline well before the stellar bar buckles, and for $f=0.3$ the gas bar declines despite the fact that the stellar bar does not seem to buckle at all.
A more detailed picture of the bar strength and length can be obtained from measurements of the profiles of the bar mode, as a function of the cylindrical radius, $A_{2}(R)$. A few examples of such profiles are shown in Fig. 5. In rows, we plotted the profiles at different times, $t=4$ and $10 \mathrm{Gyr}$, and in columns the results for stars (left column) and gas (right column) are shown. These measurements confirm that at $4 \mathrm{Gyr}$, the stellar bar is indeed the strongest for the galaxy with a gas fraction of $f=0.1$ and the gas bars are similar in all simulations. Instead, at the end of the evolution, the stellar bar is almost identical for $f=0$ and $f=$ 0.1 , a little weaker for $f=0.2$, and even more so for $f=0.3$. The gas bars are almost gone by then since $A_{2}(R)$ do not exceed 0.2 at any radius, but they are still a bit stronger and longer for lower gas fractions.

The profiles shown in Fig. 5 can also be used to estimate the length of the bars. A common way to do this is to estimate the radius where the $A_{2}(R)$ value drops to half the maximum of the profile. We thus note that at the end of the evolution, the bars in both components are significantly shorter for higher gas fractions.

A full picture of the bar evolution is presented in Fig. 6, which combines the dependence of $A_{2}$ on both the radius and time. All stellar bars start to grow around $2 \mathrm{Gyr}$, in the sense that around that time their $A_{2}$ profiles cross the threshold of 0.1 for the first time, although this happens a little earlier for the galaxy with a gas fraction of $f=0.1$. Interestingly, small perturbations in the gas component tend to appear earlier, already around $t=0.5 \mathrm{Gyr}$ in all cases. They increase particularly fast in the $f=0.1$ case and may provide the seed for the stellar bar, thus explaining its faster growth in this simulation. The effect seems to also be present for the higher gas fractions $f=0.2$ and 0.3 since these bars also start to form earlier than the one with $f=0$, although the fluctuations in the gas distribution are weaker than for $f=0.1$.

Signatures of buckling are also clearly present in these images. The stellar bars grow in strength and length until they suddenly weaken and shorten due to buckling. This happens around $6.3,4.5$, and $6.7 \mathrm{Gyr}$ for $f=0,0.1$, and 0.2 respectively, so this is significantly earlier for $f=0.1$ with respect to the other two cases as a result of faster bar growth in this case. However, no such event is visible for the largest gas fraction $f=0.3$. This bar grows steadily until about $6 \mathrm{Gyr}$ and then remains approximately the same until the end of evolution. Instead, the stellar bars in the lower gas fraction cases all start to grow again in length and strength after the buckling event.

One more difference between the stellar and gaseous bars, in addition to their different lifetimes, is their shape. As can be seen in Figs. 5 and 6 for the gas component, the profiles $A_{2}(R)$ grow much more slowly in radius compared to the stars. This is also seen in the images of the bars shown in Fig. 2: the contours of the gas bars, even at their strongest, are much more circular in the center than those in the stellar bars, which are elongated. They become more and more circular in time at larger radii, leading to the transformation into oval shapes rather than bars at the end of evolution.

As far as the pattern speeds of the bars are concerned, they are similar in all cases: for stellar and gas bars and in simulations with different gas fractions. Their values are always around $\Omega_{\mathrm{p}}=20 \mathrm{~km} \mathrm{~s}^{-1} \mathrm{kpc}^{-1}$ at the time of bar formation (defined as the moment of crossing the threshold of $A_{2}(R)=0.2$ for the first time) and they decrease down to about $10 \mathrm{~km} \mathrm{~s}^{-1} \mathrm{kpc}^{-1}$ at $10 \mathrm{Gyr}$.

Another pronounced morphological feature present in the images of Fig. 6 beside the bar is the branch of increased $A_{2}(t, R)$ values extending from the time-radius position $(t, R)=(4 \mathrm{Gyr}$, 
E. L. Łokas: The effect of warm gas on the buckling instability in galactic bars
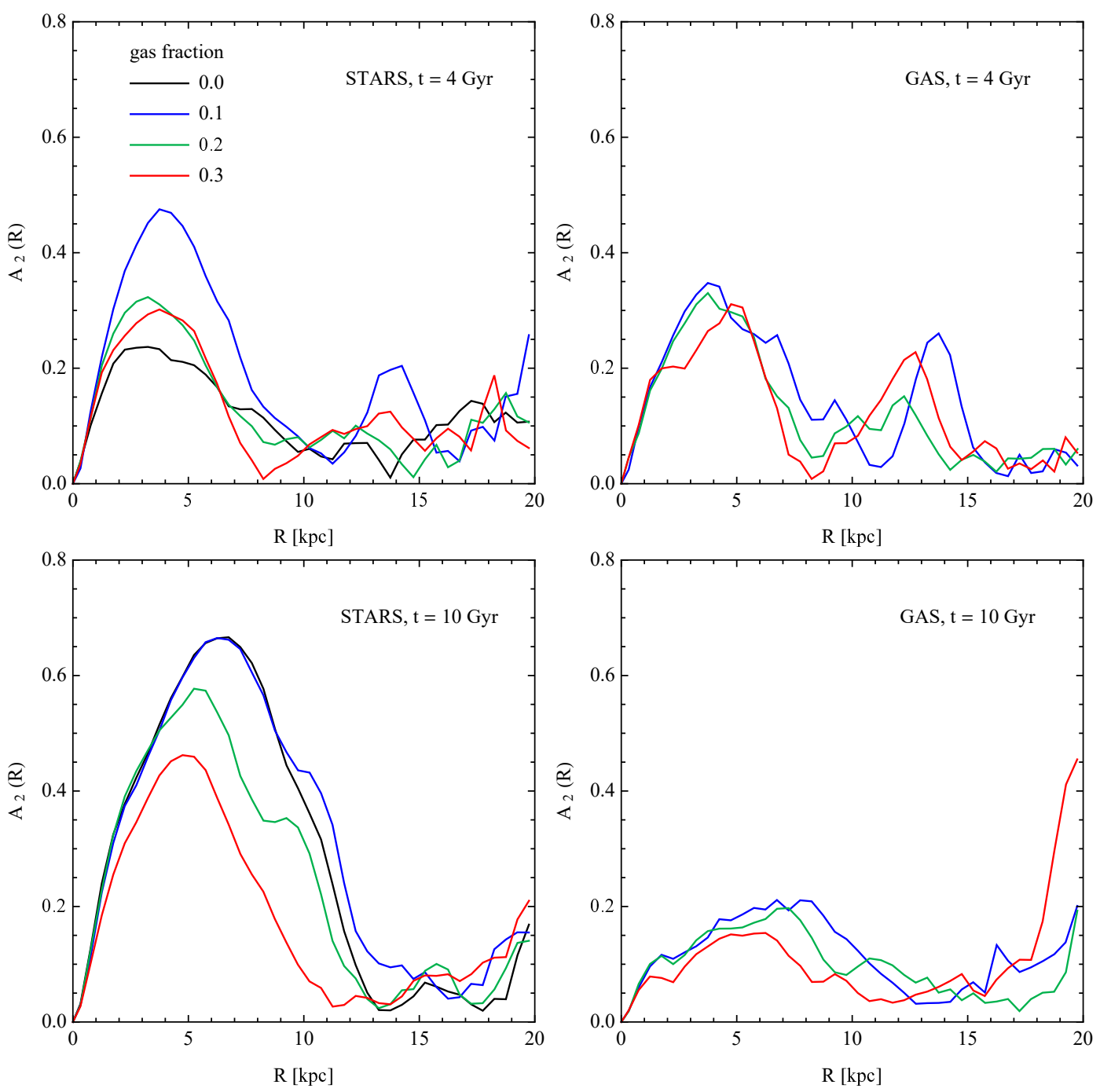

Fig. 5. Profiles of the bar mode $A_{2}(R)$ for the stars (left column plots) and gas (right column). Rows correspond to different stages of evolution: after $4 \mathrm{Gyr}$ (upper row) and after $10 \mathrm{Gyr}$ (lower row). Lines of different colors show results for different gas fractions.

$12 \mathrm{kpc})$ to $(10 \mathrm{Gyr}, 20 \mathrm{kpc})$, which is present in all panels at approximately the same location and is even stronger in gas than in stars. This feature reflects the presence of an almost ring-like, two-armed spiral structure with a very small pitch angle, forming at the corotation radius. Its increasing radius is due to the fact that while the mass distribution in the galaxies does not strongly evolve with time, the pattern speed of the bar decreases. The corotation radius, where the circular frequency equals the pattern speed, $\Omega=\Omega_{\mathrm{p}}$, thus increases with time from around $10 \mathrm{kpc}$ at the time of the formation of the bar up to $20 \mathrm{kpc}$ at $10 \mathrm{Gyr}$.

\section{Buckling instability}

In addition to the telltale signatures in the form of the weakening of the bar, the presence and effects of buckling can also be detected by measurements of the evolution of the shape of the stellar or gaseous component and the kinematics of the stars. Buckling is expected to thicken the bar significantly and this is indeed what we see in Fig. 7, showing the evolution of the ratio of the shortest to longest axis $c / a$ of the distribution of stars and gas, which is a measure of the ratio of the vertical thickness to the radial size of the bar. For gas fractions $f=0$ and 0.1 , the values of $c / a$ for the stars (upper panel) show a sharp increase at the same times as when the bars were weakened, which we identified as the times of buckling events. The increase is also present for $f=0.2$, but it is much milder. For $f=0.3$, the increase is very slow and no specific upturn is seen. The gas thickens even more, and this occurs at around the same time as for the stars. However, the thickening in the gas happens more slowly in time, so it seems as though the gas is just responding to what is happening to the stars, rather than buckling itself.

The evolution of the kinematics of the stars confirms this picture. Figure 8 shows the velocity dispersion of the stars along the cylindrical radius (upper panel), along the vertical direction (middle panel), and the ratio of the two (lower panel). While the evolution of the radial velocity dispersion reflects the evolution of the bar strength (the stronger the bar, the more radial the stellar orbits or the more stars on radial orbits), the vertical dispersion detects buckling by measuring the increase of stellar velocities in the direction perpendicular to the disk. As in the case of thickness, the simulations with low gas fractions show a sharp increase in this dispersion in contrast to the cases with higher gas fractions. The effect is even more pronounced in the evolution of the ratio of the two dispersions. We note that the minimum value of the ratio just before buckling is different in different simulations, so it is unlikely that the instability is triggered by the ratio decreasing below some characteristic 
STARS, gas fraction 0.0

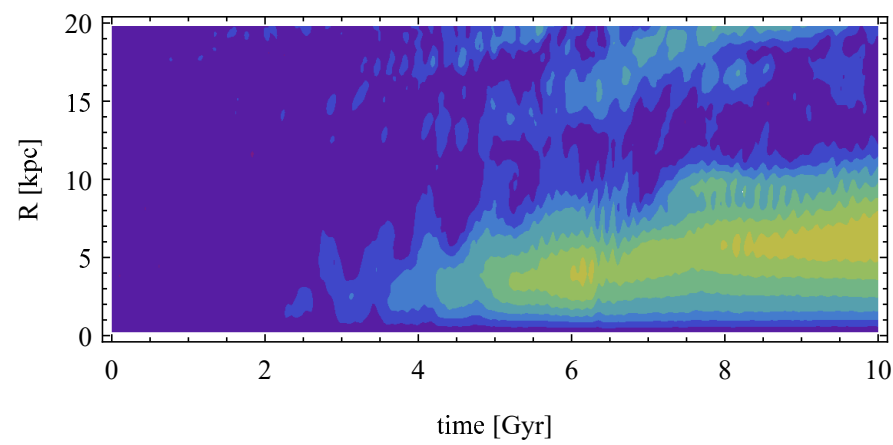

STARS, gas fraction 0.1

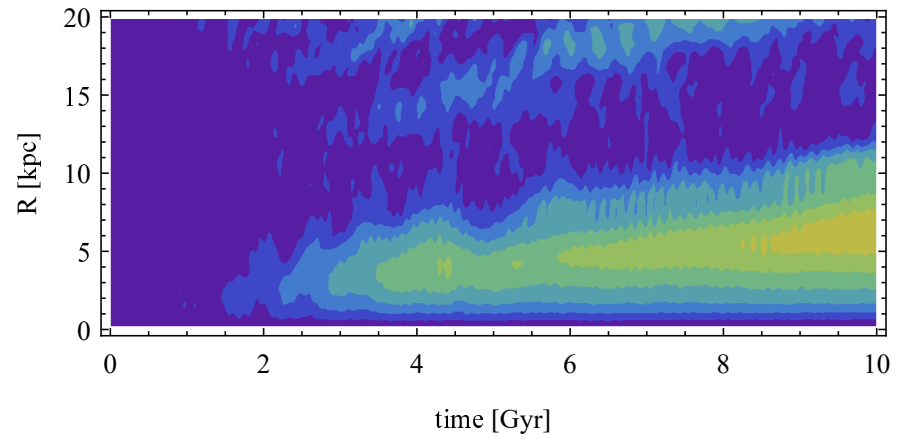

STARS, gas fraction 0.2

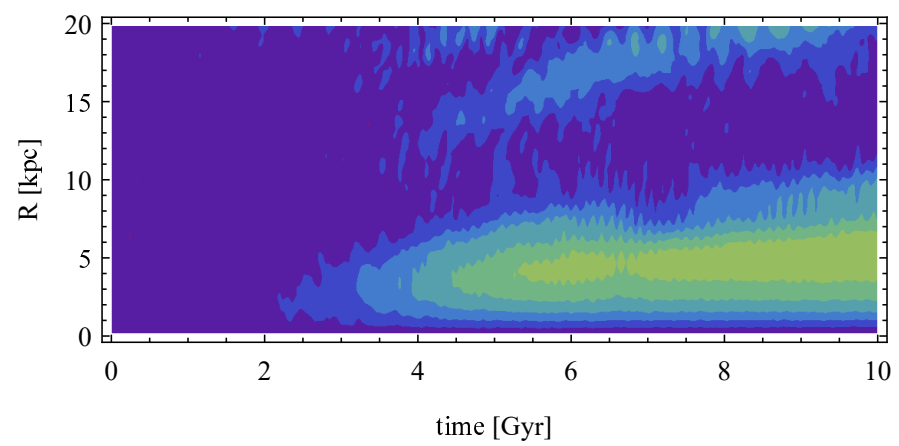

STARS, gas fraction 0.3

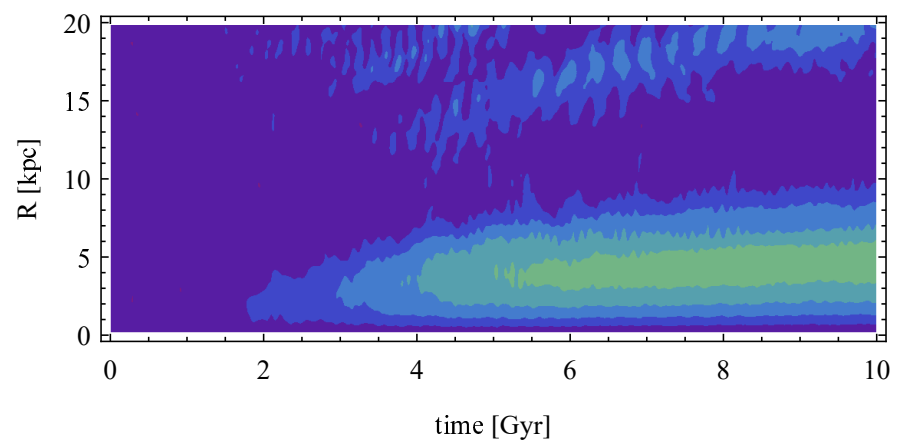

$0 \quad$ bar mode strength $\mathrm{A}_{2} \quad 1$

GAS, gas fraction 0.1

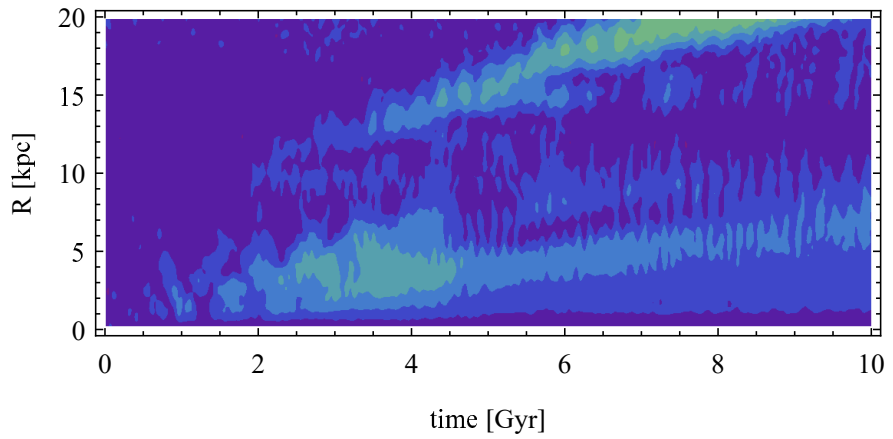

GAS, gas fraction 0.2

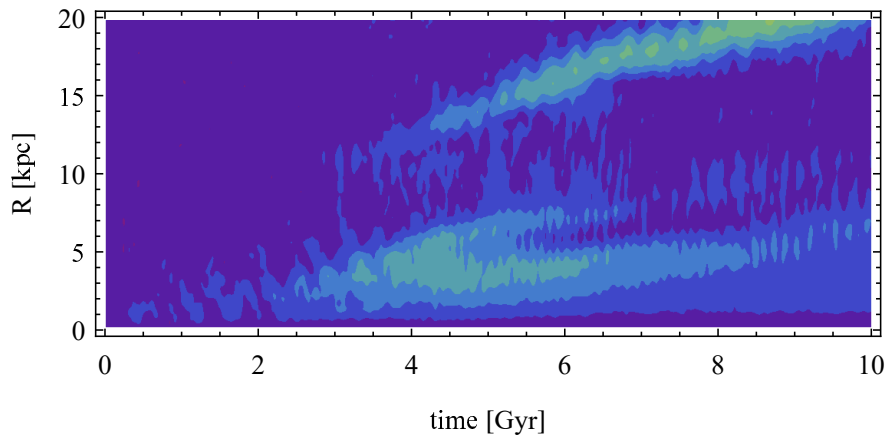

GAS, gas fraction 0.3

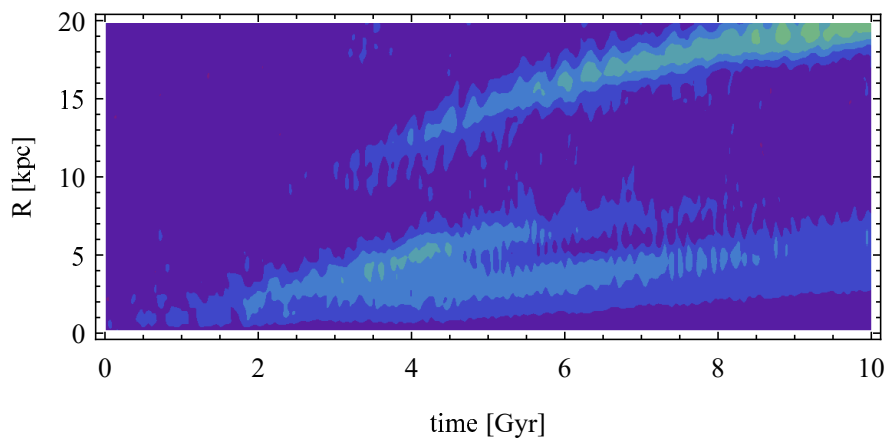

Fig. 6. Evolution of the bar mode profiles $A_{2}(R)$ in time. Left column: results for stars, right column: results for the gas. Rows from top to bottom correspond to increasing gas fraction in the galaxies.

threshold as required by the interpretation of buckling based on the fire-hose instability.

So far we have only demonstrated that, except for the case with $f=0.3$, all our bars are weakened and thickened and their stars acquire vertical motions. However, the buckling instability is also supposed to induce temporary distortions of the bar out of the disk plane. In order to detect such a distortion, we measured the mean position of the stars along the vertical direction as a function of the cylindrical radius. Figure 9 shows these measurements for the stellar components of the galaxies for the periods between 4 and $10 \mathrm{Gyr}$, that is, when the bar is already formed in all cases. We see that the strength of the buckling depends very much on the gas fraction in the disk. The times when the distortions occur coincide with the times of sudden weakening 

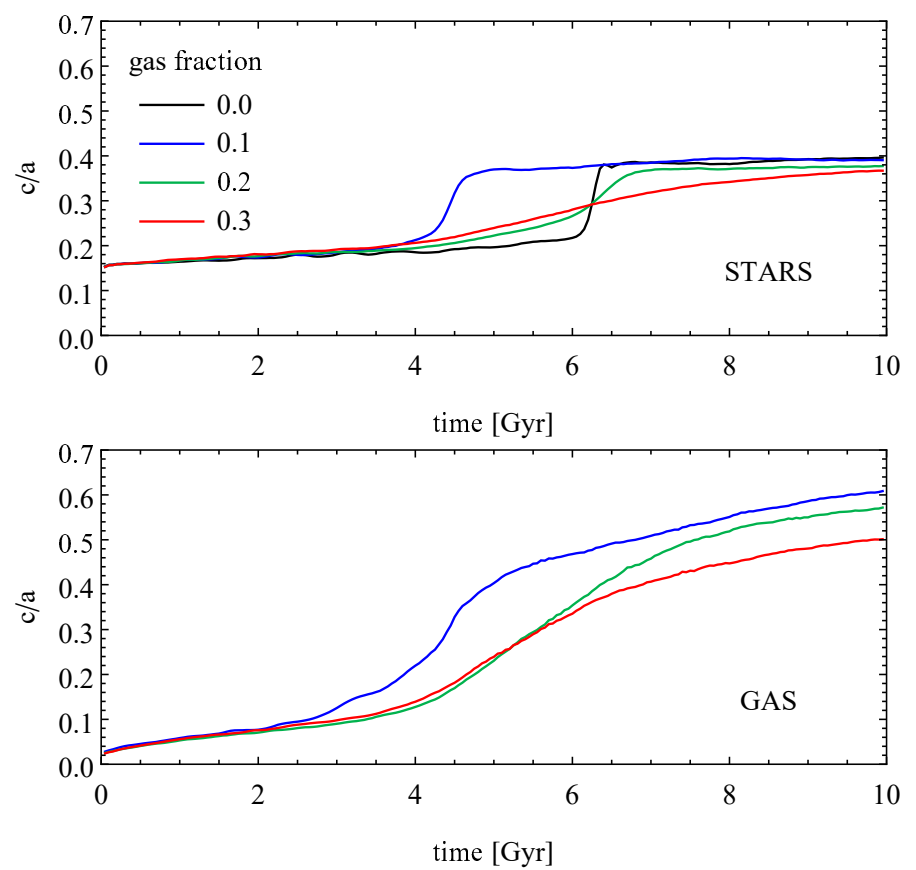

Fig. 7. Evolution of the ratio of the shortest to longest axis (the vertical thickness to the radial size of the bar) for the stars (upper panel) and gas (lower panel). Measurements were performed for particles inside $2 R_{\mathrm{D}}$. Lines of different colors correspond to different gas fractions.

and thickening of the bars discussed above, thus confirming that buckling was responsible for these events.

The distortion is clearly the strongest for the purely collisionless case with no gas (the upper panel of Fig. 9). Its shape changes from frown- to smile-like in the first phase of buckling, around $6.3 \mathrm{Gyr}$. After that, the distortion propagates outward and disappears around $7 \mathrm{Gyr}$, to reappear again a bit later in the form of secondary buckling, which lasts much longer (between 7.2 and 9 Gyr) and takes place in the outer part of the bar $(R>5 \mathrm{kpc})$.

For the simulation with a gas fraction of $f=0.1$ (the second panel of Fig. 9), the pattern is very similar, including the presence of the secondary, longer buckling phase, although the distortion is significantly weaker. It happens much earlier because the bar forms and grows earlier in this simulation. For the gas fraction 0.2 (the third panel of the figure) the signal is even weaker and more extended in time, without any clear evidence for the secondary buckling. For $f=0.3$, a very weak distortion is present within $R<8 \mathrm{kpc}$, which remains at the same level between 5 and $8 \mathrm{Gyr}$ and does not increase, so there is no clear signature of buckling in this case. For the gas component, the distortions, which are not shown here, are very weak and noisy even in the $f=0.1$ case and they are not visible at all for higher gas fractions.

The character of the distortions is illustrated further in Fig. 10 where we show in the left column the edge-on surface density distributions of the stars and in the right one the face-on maps of the distortions out of the disk plane. The rows correspond to the increasing gas fraction in the galaxies. The images are shown for the first phase of buckling, when the inner part of the bar is the most strongly distorted upward and the outer part downward. For each simulation, this occurs at a different time, so the outputs at $t=6.3,4.45,6.3$, and 5.2 were selected for simulations with a gas fraction increasing from 0 to 0.3 , respectively. We see again that the distortion weakens considerably with the increasing gas content. Although the pattern has a similar shape
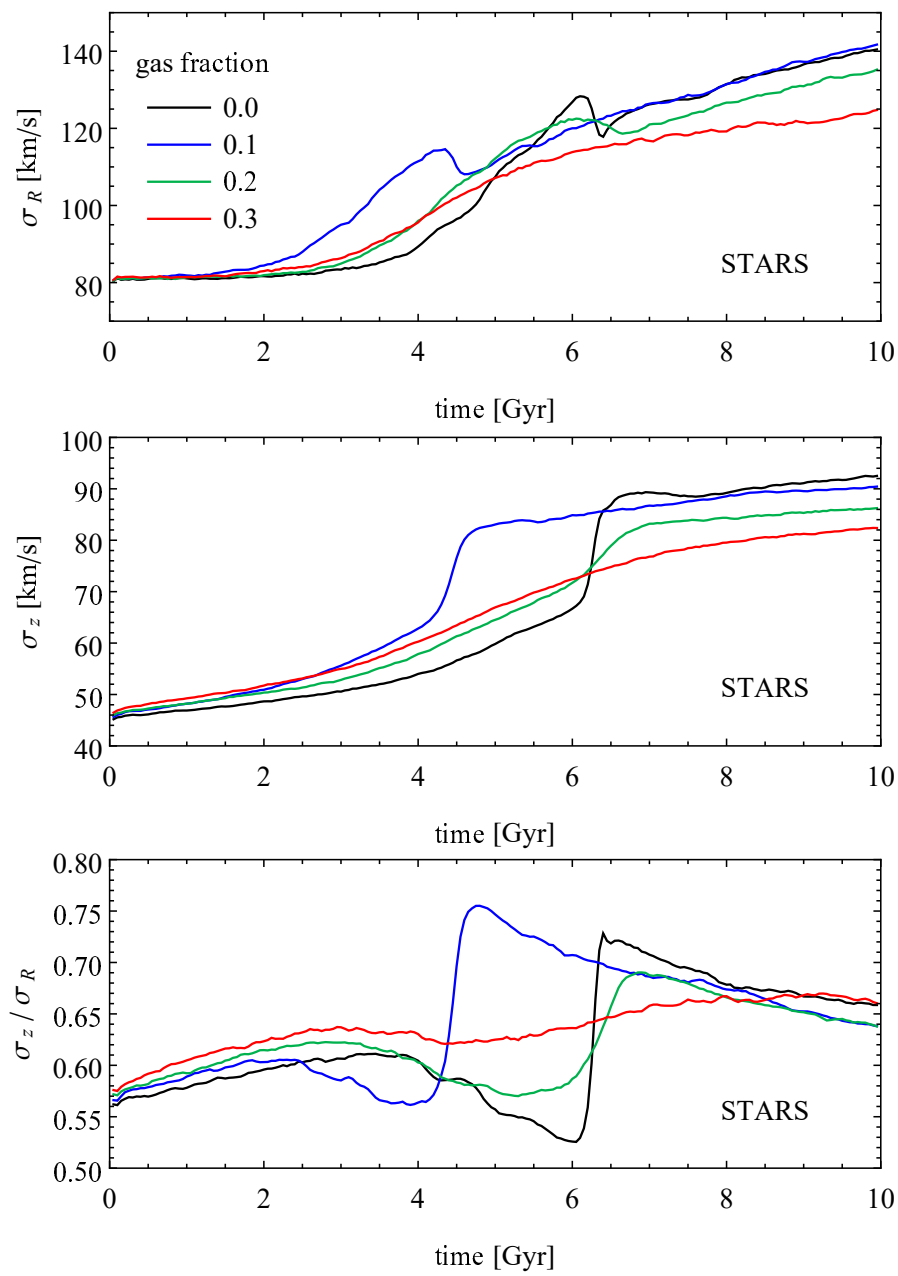

Fig. 8. Evolution of the kinematics of the stars in the form of the radial velocity dispersion (upper panel), vertical velocity dispersion (middle panel), and the ratio of the two quantities (lower panel). Measurements were performed for stars inside $2 R_{\mathrm{D}}$. Lines of different colors correspond to simulations with different gas fractions.

in each case, even for $f=0.3$, the distortion remains very weak in this case during the whole evolution. Similar patterns are also seen in the velocity distribution, which is not shown here. The subsequent evolution of the patterns is similar to the one discussed in detail in Łokas (2019b): they wind up and dissolve leaving behind bars with a distinct boxy/peanut shape.

To further explore the nature of the distortions, especially in the doubtful case of $f=0.3$, we calculated another commonly used measure of the asymmetry in the form of the $m=1$ mode of the Fourier decomposition of the surface distribution of stars in the edge-on view (projected along the intermediate $y$ axis): $A_{m z}\left(R_{x z}\right)=\left|\Sigma_{j} \exp \left(\operatorname{im} \theta_{j}\right)\right| / N$. Here, $\theta_{j}$ is the azimuthal angle of the $j$ th star and the sum is up to the total number of $N$ stars, as for the $A_{2}$ calculation, but now the radius $R_{x z}$ is measured in the $x z$ plane, $R_{x z}=\left(x^{2}+z^{2}\right)^{1 / 2}$. The $A_{1 z}\left(R_{x z}\right)$ profiles for the times where the maximum distortions occur for different simulations are shown in Fig. 11. The profiles have a similar shape for $f=0-0.2$, with a maximum within $2 R_{\mathrm{D}}$. While the shape for $f=0.3$ is different, it remains flat in the inner part of the galaxy and only increases at larger radii. This behavior confirms that in this case the buckling event did not occur.

In Fig. 12 we show the edge-on views of the galaxies at the end of evolution. The left-column plots correspond to the stellar component and the right-column ones to the gas. Clear 


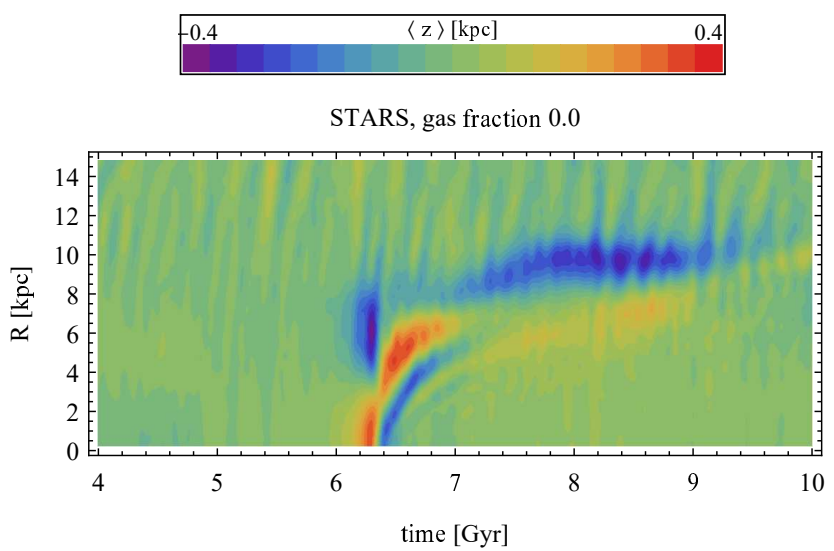

STARS, gas fraction 0.1

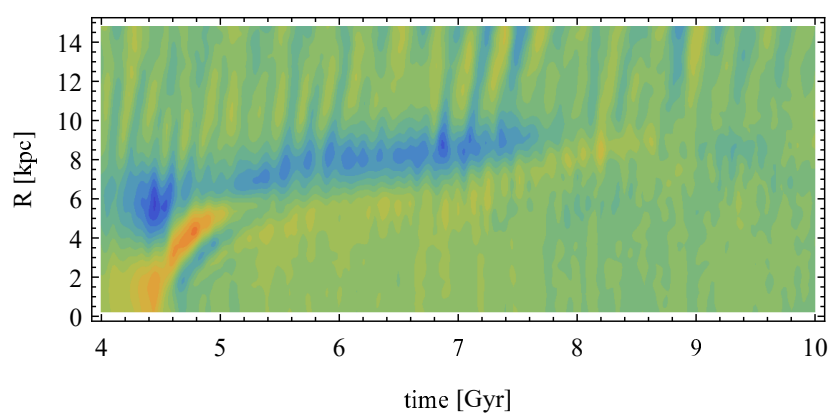

STARS, gas fraction 0.2

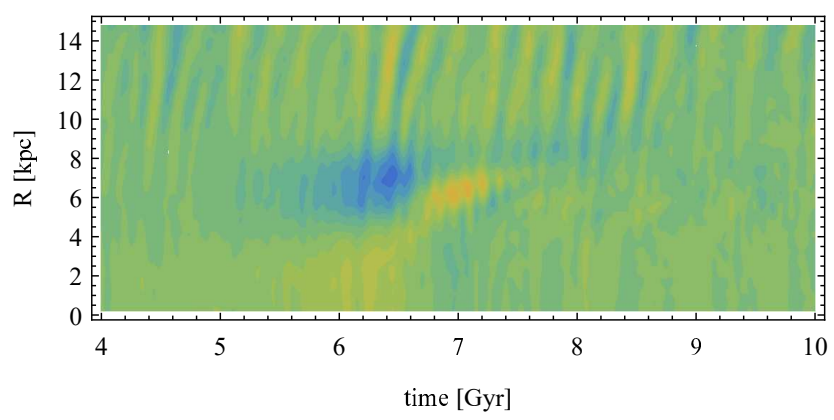

STARS, gas fraction 0.3

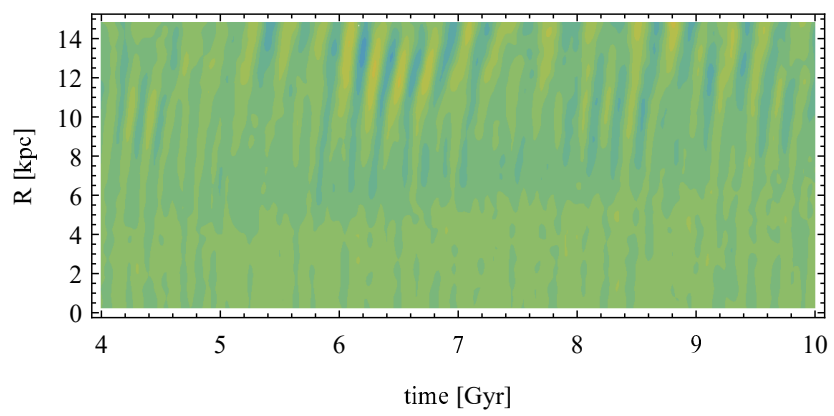

Fig. 9. Evolution of the profiles of the mean distortion of the positions of the stars along the vertical axis $z$ in simulations with different gas fractions (from top to bottom). Positive quantities point along the angular momentum vector of the disk.

boxy/peanut density distributions are present in stars as well as the gas, although their thickness decreases with an increasing gas fraction. This is even the case for the galaxy with the highest gas fraction $f=0.3$, which did not undergo buckling. Instead, the stars and gas seem to have heated vertically with an outcome that is not qualitatively different from the bars that underwent abrupt buckling events.
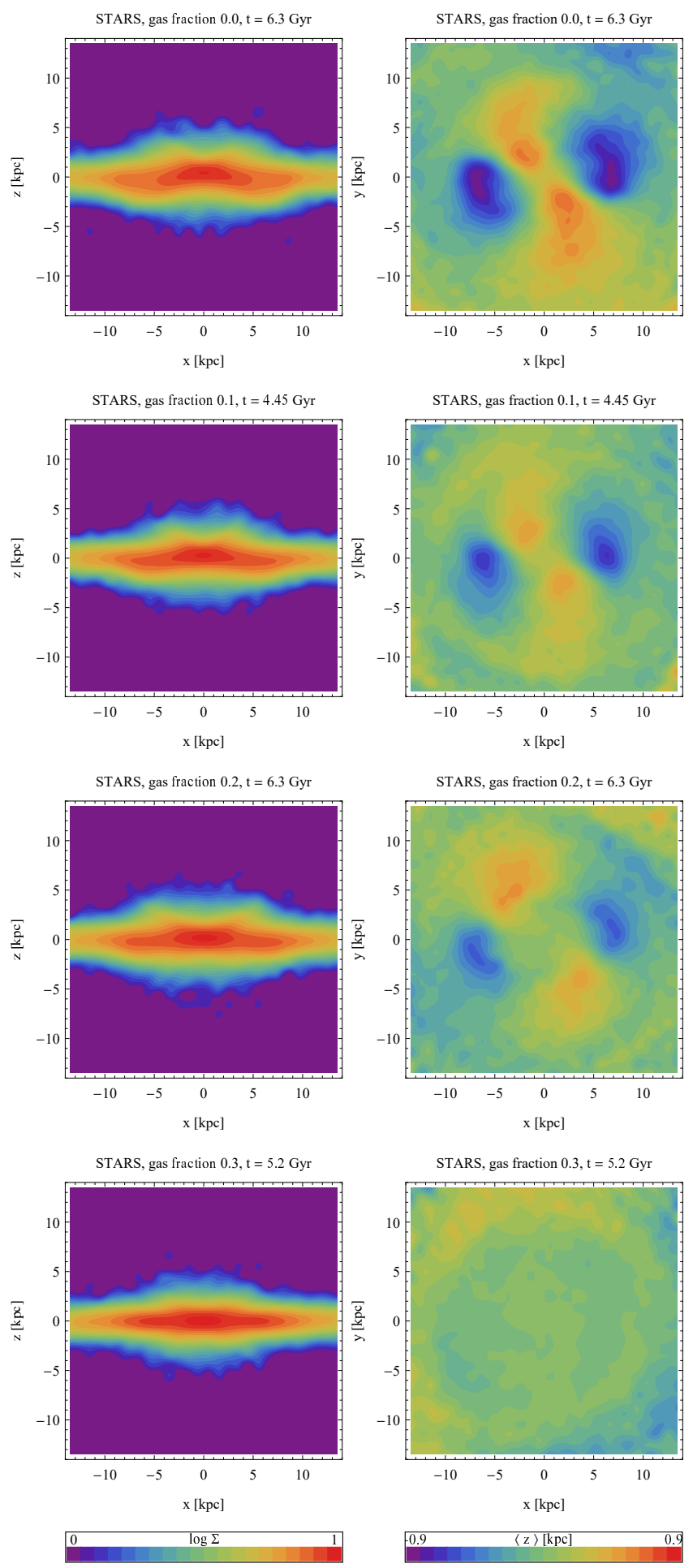

Fig. 10. Distortion of the stellar component during the first phase of buckling. Left column: surface density distributions of the stellar component viewed edge-on. The surface density was normalized to the central maximum value in each case. Right column: face-on maps of the mean distortion out of the disk plane along the vertical direction. Positive quantities point along the angular momentum vector of the disk. Rows show the results for simulations with different gas fractions.

\section{Discussion}

We studied the formation and evolution of a bar in a galaxy with parameters similar to the Milky Way evolving in isolation. The simulations differed only by the initial gas fraction, which was varied from $f=0$ to 0.3 with an increment of 0.1 . In order to 
E. L. Łokas: The effect of warm gas on the buckling instability in galactic bars

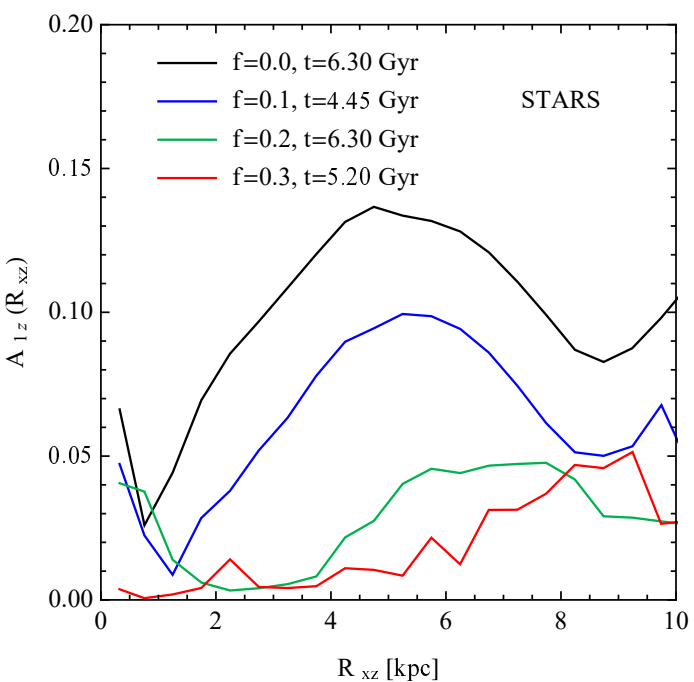

Fig. 11. Profiles of the asymmetry measure $A_{1 z}$ of the stellar component in the edge-on view during buckling.

isolate the effect of the warm gas on bar evolution, the gas fraction was kept constant during the simulations, that is, the gas was not allowed to cool and form stars. Such a configuration may reflect, to some extent, the real conditions where the gas that was already present in the disk is converted into stars, but additional gas is constantly accreted onto the disk from the hot halo of the galaxy, thus keeping the gas budget on the same level. Our main focus was to study, in detail, the effect of gas on the buckling instability in galactic bars, which has so far been mainly discussed in the context of its effect on the longevity and survival of bars.

In general, we find that bars forming in galaxies with gas are weaker than in galaxies without gas. For our particular choice of initial galaxy parameters, for gas fractions higher than 0.3 the bar did not form at all. The exact value of this threshold obviously depends on the disk susceptibility to bar formation, which is controlled by a number of parameters including the disk mass and velocity dispersion. However, the trend of weaker bars for higher gas fractions seems general and our results here agree with those of Athanassoula et al. (2013). They find that for higher gas fractions, the bars form later and grow more slowly. However, a direct comparison with our results is not possible since in their simulations, the gas fractions strongly decrease with time due to star formation and the absence of any external new gas supply to the disk. In our simulations, the bar formation is not slowed down by the presence of the gas. An interesting case is that of the gas fraction $f=0.1$ where the bar formation is even speeded up, probably by small fluctuations in the gas density which seed the stellar bar. But even though the stellar bar in this case has more time to grow, it ends up with almost exactly the same strength and length as our bar in the purely collisionless simulation (the lower left panel of Fig. 5).

Our results also agree with recent studies of barred galaxies in cosmological simulations. In particular, analyses of the Illustris and IllustrisTNG galaxy samples by Peschken \& Łokas (2019) and Rosas-Guevara et al. (2020), respectively, reveal that the fraction of barred galaxies is larger among gas-poor galaxies and that galaxies with stronger bars tend to have less gas than their unbarred counterparts. We note, however, that Rosas-Guevara et al. (2020) find strong bars even in galaxies dominated by the gas component in the disk.

Although the bars in the gas component start to grow earlier in general, we find that they are much weaker than the stellar
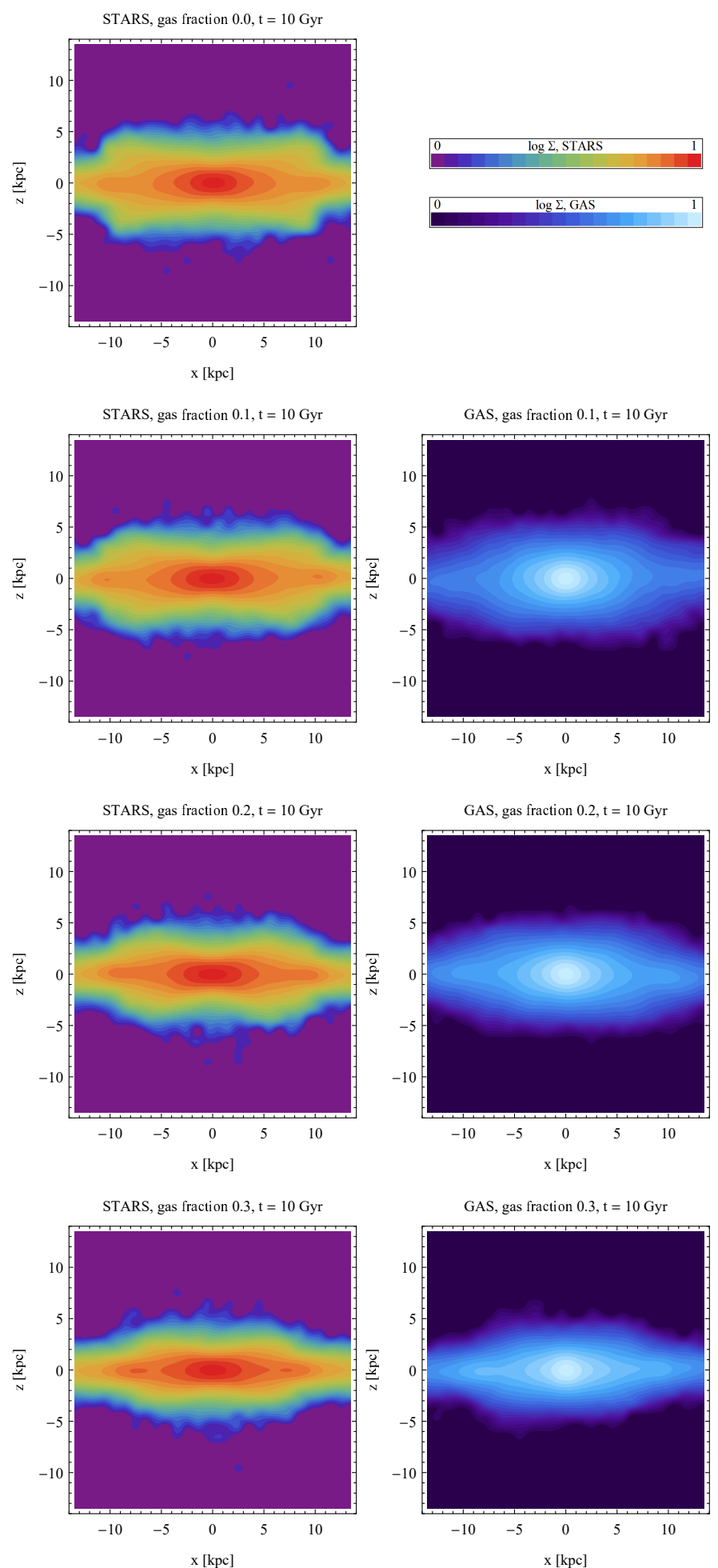

Fig. 12. Surface density distributions of stars and gas in the edge-on view at the end of the simulations, after $10 \mathrm{Gyr}$. Rows show the images from simulations with an increasing gas fraction. Left column: stars, right column: gas. The surface density was normalized to the central value in each case.

bars and reach their maximum strength around 4 Gyr. After that, they gradually weaken to acquire a spheroidal, rather than a barlike shape toward the end of the simulations. Thus we confirm that, in the words of Binney \& Tremaine (2008) "just as with humans, the dissipative lifestyle of a bar can lead to its early demise". We emphasize that this only applies to bars in the gas 
component. Once the stellar bars form, they survive until the end of evolution in good shape; they are only weaker and shorter for higher gas fractions.

Weaker stellar bars that formed with a higher gas fraction experience weaker buckling instabilities. Their distortion out of the disk plane is smaller and more extended in time. For the gas fraction $f=0.2$, the secondary phase of buckling is absent and for $f=0.3$, the buckling does not occur. Still, the process of buckling happens in a similar way in all cases: it begins with a quadrupole distortion of the bar when seen face-on, which is probably due to banana-like orbits. This pattern later winds up in the form of kinematic bending waves, as described in Łokas (2019b). All bars end up with a pronounced boxy/peanut shape when viewed edge-on at the end of simulations (Fig. 12). Interestingly, this also applies to the highest gas fraction case $(f=$ 0.3 ) where the bar did not buckle but still heated vertically, as discussed by Pfenniger \& Norman (1990) and Debattista et al. (2006). Similar shapes are visible in the gas component that did not convincingly buckle and it must have responded to the changes of the mass distribution in the stars, which was always dominant in our simulations.

Our finding that models in which buckling is weak (gas fraction $f=0.2)$ or even absent $(f=0.3)$ can still form boxy/peanut shapes agrees with the observational results of Erwin \& Debattista (2017). They find that the fraction of the boxy/peanut bulges increases strongly with the stellar mass and in the range of stellar masses considered here it depends rather weakly on the gas fraction, although no boxy/peanut shapes are found in galaxies with the present gas fraction $f>0.5$. Still, we should expect such shapes to be weaker in galaxies with higher gas fractions. The results presented here are also consistent with the recent finding of Kruk et al. (2019) who studied the fraction of galaxies with a boxy/peanut bulge as a function of redshift using data from the Hubble Space Telescope Cosmic Evolution Survey and the Sloan Digital Sky Survey. They find this fraction to strongly decrease with redshift down to zero at a redshift of $z=1$. Although no information was available concerning the gas content of higher redshift galaxies, one can expect them to be more gas-rich than their nearby counterparts. Since higher gas content can delay the vertical thickening of the bar, younger galaxies are expected to have lower boxy/peanut fractions.

Berentzen et al. (2007) only considered small gas fractions of 0.005-0.08 and did not see any clear trend of the bar strength and the strength of buckling with the increasing gas content. The only difference was a marginally shorter rise time of the bar instability in gas-richer models, which is in agreement with our result for $f=0.1$. In all of their simulations, the stellar bars grew to the same strength and this strength then dropped by the same amount. In our simulations (the upper panel of Fig. 4), this drop is smaller for higher gas fractions, thus confirming that buckling is indeed systematically weaker. They were able to detect differences in the buckling characteristics only when measuring the vertical asymmetry and thickening of the bar. Their bars only showed clear signatures of buckling asymmetry for the purely collisionless case. For the remaining gas fractions, the bars just thickened vertically retaining their vertical symmetry, which is similar to our case of $f=0.3$. We conclude that the range of gas fractions considered by Berentzen et al. (2007) was just too narrow to see a clear monotonic dependence of bar evolution and buckling on the gas fraction. While their gas fractions were motivated by the values that were actually observed in disk galaxies today, it should be kept in mind that these fractions were probably higher in the past when the bars were formed.
A larger range of gas fractions was considered in a followup study by Villa-Vargas et al. (2010). They were able to detect clear trends in the dependence of the bar properties on the gas content, but they find that their results depend strongly on the adopted gas resolution without obvious convergence. Their bars are weaker in more gas-rich disks, which is in agreement with our findings. However, although their bar mode evolution curves have sudden drops even for high gas fractions, their asymmetry measures surprisingly do not show any buckling signals. Also in disagreement with our results, for higher gas fractions, but still as low as 0.08 , their bars strongly decline in strength during the later secular evolutionary phase, while their pattern speeds increase.

Our results are in broad agreement with the recent work of Gajda et al. (2018) who studied the formation and evolution of bars in dwarf galaxies orbiting the Milky Way. Although their initial dwarf model is stable against bar formation in isolation, the bars are tidally induced and the gas fractions considered are much higher ( 0.3 and 0.7$)$, the dependence of their properties on the gas fraction is similar. The stellar bars formed more easily and survived longer for dwarfs containing less gas. Interestingly, the gas component preserved its axisymmetry throughout the entire evolution.

Our conclusion that low gas fractions favor stronger buckling and the formation of pronounced boxy/peanut shapes also agrees with the results of recent zoom-in simulations of Milky Way-like galaxies in the cosmological context from the Auriga Project. Blázquez-Calero et al. (2020) studied 21 barred galaxies from this project and identify four cases with clear boxy/peanut shapes and two more in the buckling phase, which all have a gas content below 0.2 at a redshift of $z=0$. These galaxies were further studied by Fragkoudi et al. (2019), revealing the kinematic features characteristic of buckling bars similar to those discussed in detail in Łokas (2019b).

It remains to be investigated how the processes of star formation and feedback affect the formation of bars and their buckling if there is a supply of gas from the halo. Although the results depend on a number of parameters related to the subgrid physics, which are poorly constrained as of yet, one can expect that the evolution will be somewhere in between the cases of the constant gas fractions considered here. Since the gas will be consumed by star formation, a galaxy starting with a high gas content will increase its stellar disk mass with time and become more susceptible to the formation of a stellar bar. Thus, such a galaxy may evolve as our $f=0.3$ case initially and as our $f=0.1$ case in the later stages. The transition between the two regimes will depend on the time scales of bar formation and gas consumption.

Acknowledgements. I am grateful to N. Deg for help with the GalactICS code and to the anonymous referee for useful comments. This work was supported in part by the Polish National Science Center under grant 2013/10/A/ST9/00023.

\section{References}

Abbott, C. G., Valluri, M., Shen, J., \& Debattista, V. P. 2017, MNRAS, 470, 1526 Aguerri, J. A. L., Méndez-Abreu, J., \& Corsini, E. M. 2009, A\&A, 495, 491 Athanassoula, E. 2003, MNRAS, 341, 1179

Athanassoula, E., Machado, R. E. G., \& Rodionov, S. A. 2013, MNRAS, 429, 1949

Benítez-Llambay, A., Navarro, J. F., Frenk, C. S., \& Ludlow, A. D. 2018, MNRAS, 473, 1019

Berentzen, I., Shlosman, I., Martinez-Valpuesta, I., \& Heller, C. H. 2007, ApJ, 666, 189

Blázquez-Calero, G., Florido, E., Pérez, I., et al. 2020, MNRAS, 491, 1800

Binney, J., \& Tremaine, S. 2008, Galactic Dynamics: Second Edition (Princeton, NJ: Princeton Univ. Press) 
E. L. Łokas: The effect of warm gas on the buckling instability in galactic bars

Bournaud, F., \& Combes, F. 2002, A\&A, 392, 83

Bournaud, F., Combes, F., \& Semelin, B. 2005, MNRAS, 364, L18

Buta, R. J., Sheth, K., Athanassoula, E., et al. 2015, ApJS, 217, 32

Combes, F., \& Sanders, R. H. 1981, A\&A, 96, 164

Combes, F., Debbasch, F., Friedli, D., \& Pfenniger, D. 1990, A\&A, 233, 82

Debattista, V. P., Mayer, L., Carollo, C. M., et al. 2006, ApJ, 645, 209

Deg, N., Widrow, L. M., \& Randriamampandry, T. 2019, MNRAS, 486, 5391

Erwin, P. 2018, MNRAS, 474, 5372

Erwin, P., \& Debattista, V. P. 2017, MNRAS, 468, 2058

Fragkoudi, F., Grand, R. J. J., Pakmor, R., et al. 2019, MNRAS, submitted [arXiv:1911.06826]

Gajda, G., Łokas, E. L., \& Athanassoula, E. 2017, ApJ, 842, 56

Gajda, G., Łokas, E. L., \& Athanassoula, E. 2018, ApJ, 868, 100

Hohl, F. 1971, ApJ, 168, 343

Hopkins, P. F. 2015, MNRAS, 450, 53

Hopkins, P. F., Wetzel, A., Kereš, D., et al. 2018, MNRAS, 480, 800

Knapen, J. H., Shlosman, I., \& Peletier, R. F. 2000, ApJ, 529, 93

Kruk, S. J., Erwin, P., Debattista, V. P., \& Lintott, C. 2019, MNRAS, 490, 4721

Łokas, E. L. 2018, ApJ, 857, 6

Łokas, E. L. 2019a, A\&A, 624, A37

Łokas, E. L. 2019b, A\&A, 629, A52

Łokas, E. L., Athanassoula, E., Debattista, V. P., et al. 2014, MNRAS, 445, 1339

Łokas, E. L., Ebrová, I., del Pino, A., et al. 2016, ApJ, 826, 227

Martinez-Valpuesta, I., Shlosman, I., \& Heller, C. 2006, ApJ, 637, 214
Merritt, D., \& Hernquist, L. 1991, ApJ, 376, 439

Merritt, D., \& Sellwood, J. A. 1994, ApJ, 425, 551

Nair, P. B., \& Abraham, R. G. 2010, ApJ, 714, L260

Navarro, J. F., Frenk, C. S., \& White, S. D. M. 1997, ApJ, 490, 493

Noguchi, M. 1996, ApJ, 469, 605

Ostriker, J. P., \& Peebles, P. J. E. 1973, ApJ, 186, 467

Peschken, N., \& Łokas, E. L. 2019, MNRAS, 483, 2721

Pfenniger, D., \& Friedli, D. 1991, A\&A, 252, 75

Pfenniger, D., \& Norman, C. 1990, ApJ, 363, 391

Portail, M., Wegg, C., \& Gerhard, O. 2015, MNRAS, 450, L66

Quillen, A. C. 2002, AJ, 124, 722

Raha, N., Sellwood, J. A., James, R. A., \& Kahn, F. D. 1991, Nature, 352, 411

Rosas-Guevara, Y., Bonoli, S., Dotti, M., et al. 2020, MNRAS, 491, 2547

Roškar, R., Debattista, V. P., Stinson, G. S., et al. 2008, ApJ, 675, L65

Sellwood, J. A. 1981, A\&A, 99, 362

Sellwood, J. A., \& Debattista, V. P. 2009, MNRAS, 398, 1279

Springel, V. 2005, MNRAS, 364, 1105

Springel, V., Yoshida, N., \& White, S. D. M. 2001, New Astron., 6, 79

Toomre, A. 1966, Geophys. Fluid Dyn., 66-46, 111

Villa-Vargas, J., Shlosman, I., \& Heller, C. 2010, ApJ, 719, 1470

Wang, H.-H., Klessen, R. S., Dullemond, C. P., van den Bosch, F. C., \& Fuchs, B. 2010, MNRAS, 407, 705

Widrow, L. M., \& Dubinski, J. 2005, ApJ, 631, 838

Widrow, L. M., Pym, B., \& Dubinski, J. 2008, ApJ, 679, 1239 